\title{
EQUILIBRIUM STUDY BETWEEN CONDENSED PHASES BY ISOPLETHIC THERMAL ANALYSIS WHEN A MISCIBILITY GAP IS OBSERVED
}

\author{
E. Labarthe, A.J. Bougrine, H. Delalu, J. Berthet and J.J. Counioux
}

Université de Lyon, Université Lyon 1, Laboratoire Hydrazines et Procédés - UMR CNRS 5179, F-69622 Villeurbanne Cedex

\begin{abstract}
Isoplethic thermal analysis was used to determine the solid-liquid-liquid equilibria in the ternary system water-sodium sulfatepiperidine. The changes in state observed on the thermogram recorded during the displacement in a quasi-binary section permit the identification of the different phases and the delimitation of the corresponding equilibrium domains. Two isotherms were established at $25^{\circ} \mathrm{C}$ and $40^{\circ} \mathrm{C}$ because these temperatures frame the peritectic decomposition of the sodium sulfate decahydrate. Miscibility gaps were completely delimited and each critical point was calculated. This study permitted to determine the optimal conditions for separating the organic phase by liquid-liquid extraction.
\end{abstract}

Key words: ternary diagram, isoplethic thermal analysis, miscibility gap, critical point, piperidine, water, sodium sulfate

\section{Introduction}

Studying a high order system requires long and painstaking experimentations when using traditional analysis techniques. Therefore, the laboratory has developed automated isoplethic methods that consist in progressively modifying the global composition of the system, monitoring the evolution of an intensive variable and using the information obtained. The first works ${ }^{[1]}$, which were based on successive and then continuous conductimetric measurements, demonstrated how it was possible to determine all the characteristics of an isotherm: solubility curves, invariant points, nature and composition of solid phases.

Direct or differential thermal analysis rests on the exploitation of the thermal effects that occur when a sample is subjected to a regular variation of its heat energy. The progressive addition of a given solution in a mixture modifies its global composition. The molar and mass compositions of each constituent vary linearly through time and thus define an isoplethic section of the system formed by all the independent constituents. Isoplethic Thermal Analysis ${ }^{[2-3]}$, or ITA, consists in using the thermal phenomena observed under almost constant pressure and temperature conditions.

\section{Experimental}

In an invariant isotherm-isobar system, the number of phases in equilibrium is equal to the number of independent constituents $\mathrm{c}$. If an infinitesimal quantity da (molar or mass unit) of a mixture A, with composition vector $\vec{A}$, is added to an invariant system composed of c phases $\mathrm{j}$ of composition vector $\overrightarrow{F_{j}}$ in quantity $\mathrm{f}_{\mathrm{j}}$, only the quantities $d f_{j}$ of these phases will be modified. According to the properties of the isobarycentric transformations ${ }^{[4]}$, these thermodynamic variables satisfy the relation (1):

$$
d a \cdot \vec{A}=\sum_{j=1}^{j=c} d f_{j} \cdot \overrightarrow{F_{j}}
$$

which can also be written as:

$$
\sum_{j=1}^{j=c} d f_{j} \cdot \overrightarrow{A F_{j}}=\overrightarrow{0}
$$

since $\sum_{j=1}^{j=c} d f_{j}=d a$

The resolution of this linear equation system permits the calculation of each $d f_{j}$ for a given value of $d a$. If $H_{A}$ is the enthalpy of the mixture added and $H_{j}$ is the enthalpy of the phase $\mathrm{j}$, the enthalpy difference (molar or in mass) of the system is given by:

$$
d H=\sum_{j=1}^{j=c} d f_{j} \cdot H_{j}-d a \cdot H_{A}
$$

In the case of an invariant system, $d H$ depends only on the added quantity $d a$. In particular, this variation of enthalpy is not a function of the mass and composition of the initial system but it is directly proportional to $d a$.

If the mixture $\mathrm{A}$ is introduced at a constant flow rate, $q=d a / d t$, the heat quantity involved per time unit is constant:

$$
d H / d t=k_{1} \cdot q
$$

In addition, if the measurement cell is of isoperibolic type, the heat flow $\Phi$, to which the 
system is subjected, is proportional to the variation of temperature, according to Newton's law:

$$
\Phi=\frac{\delta Q}{d t}=k_{2} . \Delta T
$$

Where $\Delta T$ is the temperature difference between the mixture temperature $T_{M}$ and the thermostatic chamber temperature $T_{C}$.

$$
\Delta T=T_{M}-T_{C}
$$

Hence the relation:

$$
\Delta T=\left(k_{1} / k_{2}\right) \cdot q=K \cdot q
$$

Finally, for an invariant isotherm-isobar system, a given mixture and injection speed, the variation of temperature between the mixture and the thermostatic chamber is constant and a temperature plateau should be observed. The displacement of an indifferent equilibrium is therefore expressed by ITA as a plateau of invariance, as in direct thermal analysis.

In practice, the variation of the temperature $\Delta \mathrm{T}$ is recorded as a function of the volume $\mathrm{v}$ of solution added. Each equilibrium domain has a corresponding specific variation law, allowing the determination of phase transformations.

An experimental set-up was built ${ }^{[5]}$ according to the principle defined previously and adjusted in particular to a temperature measurement sensitivity in the region of $\mathrm{mK}$. This set-up is completely automated and is composed of a platinum probe Pt100, a thermostat Hetofrig type CB11, a reactor, an automatic injection system and a magnetic agitator.

The injection of the solvent in the stirred mixture is performed by a computer controlled automatic injection system. The injection range used is within 1 and $4 \mathrm{~mL} /$ hour. The temperature values are stored at regular time intervals.

The phase transformations are determined by computer processing of the numerical data by linear extrapolation of the variation laws.

This device was applied to the study of equilibria between the solid-liquid phases and liquid-liquid phases of the ternary system watersodium sulfate-piperidine, used as the basis for extracting organic compounds in a complex synthetic mixture.

The ternary system studied is delimited by three binary systems. The $\mathrm{Na}_{2} \mathrm{SO}_{4}-\mathrm{C}_{5} \mathrm{H}_{10} \mathrm{NH}$ system is unknown, while in the temperature field studied the piperidine is totally miscible in water. On the other hand, two hydrates are present in the binary mixture $\mathrm{H}_{2} \mathrm{O}-\mathrm{Na}_{2} \mathrm{SO}_{4}{ }^{[6-7]}$ : a metastable heptahydrate and a stable decahydrate (Glauber salt) which undergoes a peritectic decomposition at $32.4^{\circ} \mathrm{C}$.

In order to analyse the impact of the decahydrate on the equilibria, we established two isotherms at $25^{\circ} \mathrm{C}$ and $40^{\circ} \mathrm{C}$, that frame its decomposition temperature.

Figure 2 gives an example of a thermogram obtained from a ternary mixture whose composition point $\mathrm{A}$ is located in Figure 3. It shows the evolution of the temperature mixture when a volume of pure water is added.

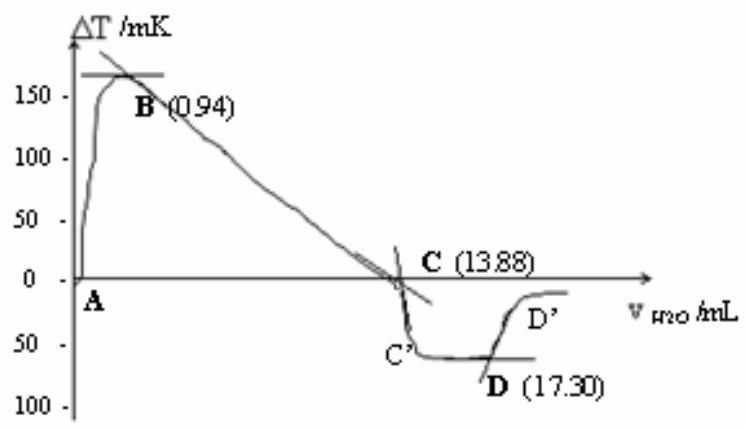

Fig.1:Example of thermogram registred during addition of pure water in the ternary mixture A.

Segment $A B$ corresponds to the progressive hydration of anhydrous sodium sulfate into decahydrated salt. Reaching steady state was terminated with a short plateau of invariance. From $\mathrm{B}$ to $\mathrm{C}$, crossing the two phases equilibrium domain liquid $+\mathrm{Na}_{2} \mathrm{SO}_{4} \cdot 10 \mathrm{H}_{2} \mathrm{O}$ is monotonous. After reaching steady state $\mathrm{C}$ at $\mathrm{C}^{\prime}$, crossing the triphasic domain $\mathrm{L} 1+\mathrm{L} 2+\mathrm{Na}_{2} \mathrm{SO}_{4} \cdot 10 \mathrm{H}_{2} \mathrm{O}$ is shown by a plateau of invariance up to point $\mathrm{D}$. Point $\mathrm{D}$, beyond which no solid phase can be observed, corresponds to the limit conode of the miscibility gap. By using this technique, the different equilibrium domains are clearly defined and permit the full determination of the isotherm.

\section{Results and discussion}

Figures 2 and 3 represent isotherms $25^{\circ} \mathrm{C}$ and $40^{\circ} \mathrm{C}$ respectively of the ternary system $\mathrm{H}_{2} \mathrm{O}-\mathrm{Na}_{2} \mathrm{SO}_{4}$ $\mathrm{C}_{5} \mathrm{H}_{10} \mathrm{NH}$ while tables 1 and 2 give the associated experimental values, expressed in mass fractions. 


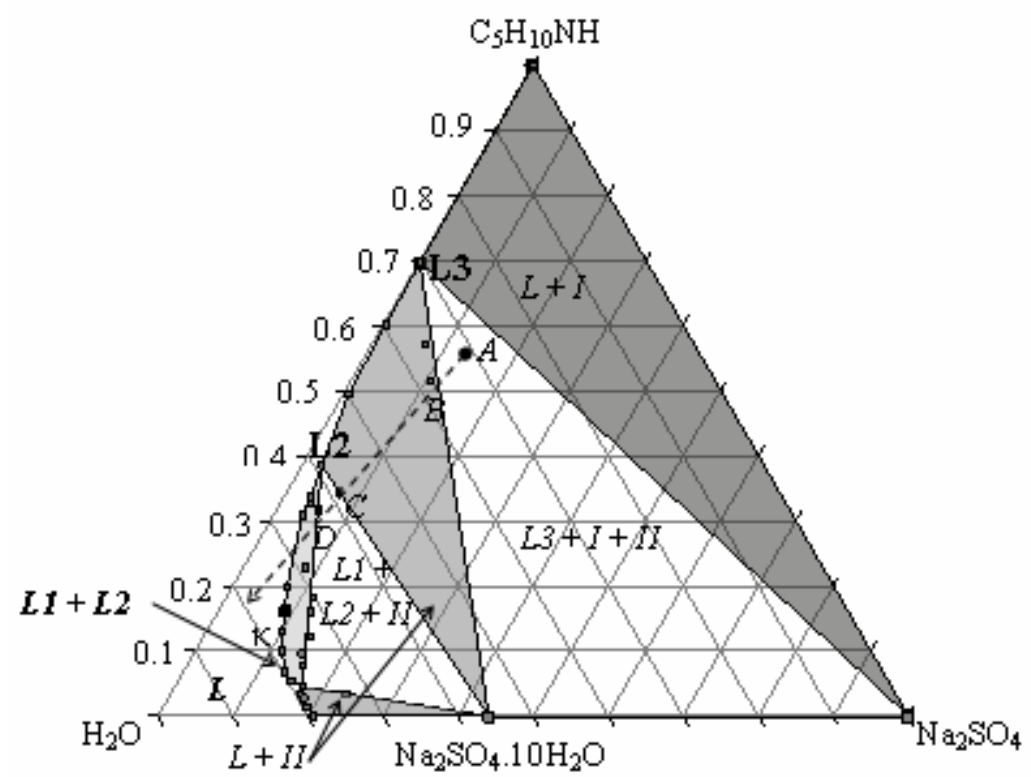

Fig.2: Ternary system $\mathrm{H}_{2} \mathrm{O}-\mathrm{Na}_{2} \mathrm{SO}_{4}-\mathrm{C}_{5} \mathrm{H}_{10} \mathrm{NH}$ : isotherm $25^{\circ} \mathrm{C}$ (mass fractions) Solid phase I: $\mathrm{Na}_{2} \mathrm{SO}_{4}$

Solid phase II: $\mathrm{Na}_{2} \mathrm{SO}_{4} \cdot 10 \mathrm{H}_{2} \mathrm{O}$

\begin{tabular}{|c|c|c|}
\hline $\mathrm{w}\left(\mathrm{C}_{5} \mathrm{H}_{10} \mathrm{NH}\right)$ & $\mathrm{w}\left(\mathrm{Na}_{2} \mathrm{SO}_{4}\right)$ & Phenomenon \\
\hline 0 & 0.207 & Liquidus of $\mathrm{Na}_{2} \mathrm{SO}_{4} \cdot 10 \mathrm{H}_{2} \mathrm{O}$ \\
\hline 0.0144 & 0.2011 & 1 \\
\hline 0.0272 & 0.1811 & " \\
\hline 0.0345 & 0.1731 & " \\
\hline 0.045 & 0.170 & Invariant point $\mathrm{L} 1 *$ \\
\hline 0.0350 & 0.2333 & Limit conode $\mathrm{L} 1-\mathrm{Na}_{2} \mathrm{SO}_{4} \cdot 10 \mathrm{H}_{2} \mathrm{O}$ \\
\hline 0.054 & 0.151 & $\begin{array}{l}\text { binodal } \\
\text { bister }\end{array}$ \\
\hline 0.0680 & 0.136 & " \\
\hline 0.0995 & 0.1161 & " \\
\hline 0.1304 & 0.1012 & " \\
\hline 0.16 & 0.09 & Critical point $\kappa^{* *}$ \\
\hline 0.198 & 0.073 & Critical point $\mathrm{K}^{\mathrm{*}}$ \\
\hline 0.3085 & 0.0383 & oinodal \\
\hline 0.336 & 0.036 & I imit conode I 1-I 2 \\
\hline 0.0770 & 0.1539 & " \\
\hline 0.0956 & 0.1439 & " \\
\hline 0.1214 & 0.1417 & $"$ \\
\hline 0.1602 & 0.1243 & " \\
\hline 0.1830 & 0.1161 & " \\
\hline 0.2273 & 0.0835 & 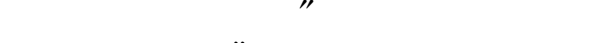 \\
\hline 0.3160 & 0.0551 & " (point D) \\
\hline 0.3267 & 0.0406 & " \\
\hline 0.385 & 0.026 & Invariant point $\mathrm{L} 2 *$ \\
\hline 0.3438 & 0.0708 & Limit conode $\mathrm{L} 2-\mathrm{Na}_{2} \mathrm{SO}_{4} \cdot 10 \mathrm{H}_{2} \mathrm{O}$ (point $\mathrm{C}$ ) \\
\hline 0.495 & 0.0243 & Liquidus of $\mathrm{Na}_{2} \mathrm{SO}_{4} \cdot 10 \mathrm{H}_{2} \mathrm{O}$ \\
\hline 0.60 & 0.0039 & " \\
\hline 0.5694 & 0.0711 & Limit conode $\mathrm{L} 3-\mathrm{Na}_{2} \mathrm{SO}_{4} \cdot 10 \mathrm{H}_{2} \mathrm{O}$ \\
\hline 0.5152 & 0.1061 & " (point B) \\
\hline 0.67 & $<0.001$ & Invariant point $\mathrm{L} 3 *$ \\
\hline
\end{tabular}

Table 1: Isotherm $25^{\circ} \mathrm{Cdetermined} \mathrm{by} \mathrm{ATI,} \mathrm{chemical} \mathrm{analysis}(*)$ or generalized diameter method (**)

The $25^{\circ} \mathrm{C}$ isotherm is characterized by the crystallization of two solid phases, the anhydrous and the sodium sulfate decahydrate, and the existence of a miscibility gap in the crystallization area of the decahydrate. The liquid-liquid equilibrium is delimited by the conode L1-L2 and the critical point $\kappa$. The solubility of the anhydrous sulfate in concentrated aqueous solutions of piperidine is very weak and the liquidus curve is 
almost confounded with the limit binary system

$\mathrm{H}_{2} \mathrm{O}-\mathrm{C}_{5} \mathrm{H}_{10} \mathrm{NH}$.

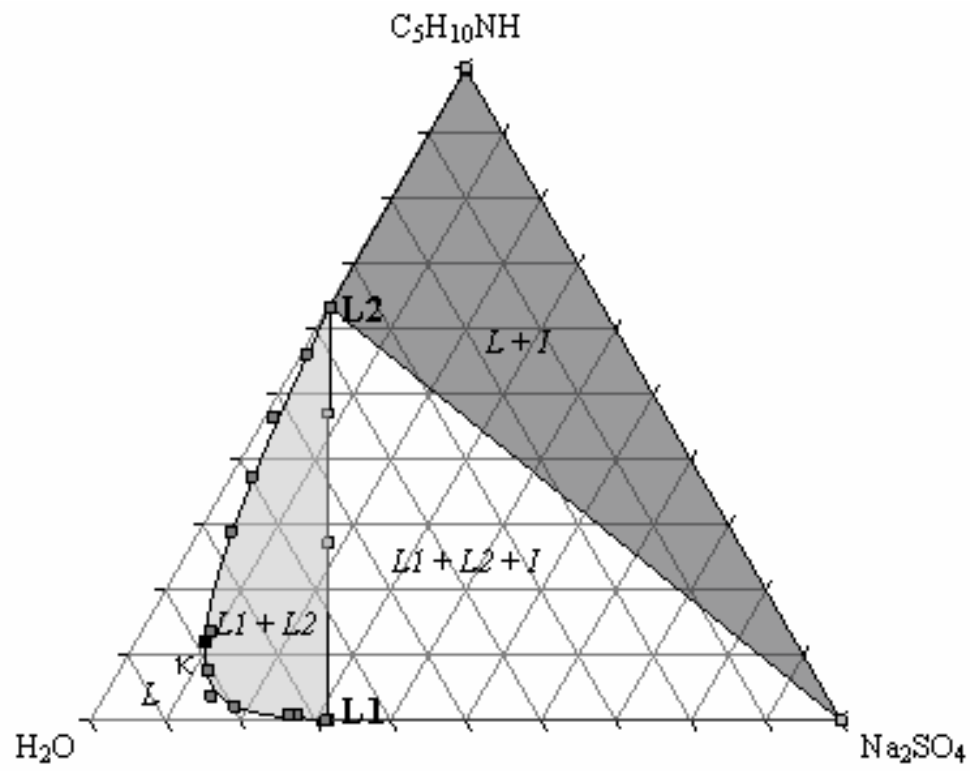

Fig.3: Ternary system $\mathrm{H}_{2} \mathrm{O}-\mathrm{Na}_{2} \mathrm{SO}_{4}-\mathrm{C}_{5} \mathrm{H}_{10} \mathrm{NH}$ : isotherm $40^{\circ} \mathrm{C}$ (mass fractions) Solid phase I: $\mathrm{Na}_{2} \mathrm{SO}_{4}$

\begin{tabular}{|c|c|c|}
\hline $\mathrm{w}\left(\mathrm{C}_{5} \mathrm{H}_{10} \mathrm{NH}\right)$ & $\mathrm{w}\left(\mathrm{Na}_{2} \mathrm{SO}_{4}\right)$ & Phenomenon \\
\hline 0.0009 & 0.314 & Invariant point L1* \\
0.008 & 0.272 & binodal \\
0.008 & 0.259 & " \\
0.022 & 0.182 & $"$ \\
0.039 & 0.142 & " \\
0.078 & 0.118 & Critical point $\kappa^{* *}$ \\
0.12 & 0.10 & binodal \\
0.137 & 0.092 & " \\
0.291 & 0.041 & " \\
0.375 & 0.027 & " \\
0.465 & 0.011 & Limit conode L1-L2 \\
0.563 & 0.006 & " \\
0.04 & 0.30 & " \\
0.272 & 0.181 & Invariant point L2* \\
0.474 & 0.067 & \\
0.61 & 0.014 & \\
0.628 & 0.002 & \\
\hline
\end{tabular}

Table 2: Isotherm $40^{\circ} \mathrm{C}$ determined by ATI, chemical analysis $(*)$ or generalized diameter method $(* *)$

At $40^{\circ} \mathrm{C}$, the decahydrate is no more stable and the liquidus curve of the anhydrous salt is interrupted by the demixing curve. One of the parts of the liquidus is almost confounded with the point $\mathrm{L} 1$.

The liquid-liquid area is stimgly dependent on temperature. The critical line, that separates the two half surfaces of the binodal, is a fundamental characteristic of the miscibility gap. However, even at a given temperature, its composition is difficult to define experimentally. Ideally, when the two branches of the binodal curve are almost symmetrical, determining the coordinates of the critical point by using the diameter method is possible when a series of conodes is known. Its application to the two isotherms studied shows, unfortunately, that the curvatures are too important to allow reliable extrapolation and we have adapted this method to non-symmetrical curves by barycentric weighting of two liquid phases. For each conode (i), the point $\mathrm{P}_{\mathrm{i}}$ obtained satisfies the relation:

$\overrightarrow{P_{i}}=w_{1 i} \cdot \overrightarrow{L_{1 i}}+w_{2 i} \cdot \overrightarrow{L_{2 i}}$

where $\mathrm{w}_{1 \mathrm{i}}$ and $\mathrm{w}_{2 \mathrm{i}}$ represent respectively the mass fractions of the liquid phases of compositions 
$\overrightarrow{L_{1 i}}$ and $\overrightarrow{L_{2 i}}$. The correlation coefficients $\mathrm{R}^{2}$ of the regression lines are calculated as a function of the parameter $\mathrm{w}=\mathrm{w}_{2 \mathrm{i}}$, then the curve $\mathrm{R}^{2}=$ $\mathrm{f}(\mathrm{w})$ is plotted as shown in the example in Figure 4.

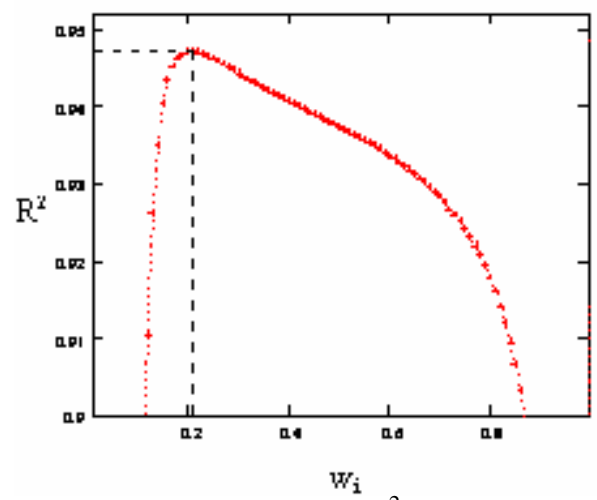

Fig.4: Correlation coefficient $\mathrm{R}^{2}$ as a function of the mass fraction of liquid phase $\left(\mathrm{L} 2_{\mathrm{i}}\right)$

The value of $\mathrm{w}$ corresponding to the best correlation coefficient is chosen to then define the coordinates of the critical point by intersection with the binodal.

\section{Conclusions}

After a theoretical analysis of the bases of the Isoplethic Thermal Analysis intended to improve the use of thermograms, this technique was applied to the study of the water-sodium sulfatepiperidine system. The two isotherms $25^{\circ} \mathrm{C}$ and $40^{\circ} \mathrm{C}$, framing the peritectic decomposition of the sodium sulfate decahydrate, were both plotted in full and, in particular, the miscibility gap which appears was completely delimited: the critical point of the binodal and the limit conode, corresponding to the appearance of anhydrous and decahydrated sodium sulfate were identified.

Lastly, the experimental results obtained constitute fundamental data for treating more or less complex solutions containing the three constituents taken into account. In particular, this study highlighted that increasing temperature favours extraction potential by liquid-liquid separation and determines the associated conditions.

\section{References}

1 R. Tenu, J.J. Counioux and R. Cohen-Adad, Bull. Soc. Chim. Fr., 3-4, I (1979), 82-85

2 R. Tenu, J.J. Counioux, Bull. Soc. Chim. 5-6 (1979) 155

3 J. Berthet, R. Tenu, J.J. Counioux, Thermochim. Acta 68 (1983) 145.

4 J. Berthet and J.J. Counioux, ISBN2-9504481-5-1, JEEP XX (March 1994)

5 J. Berthet and J.J. Counioux, Brevet F9313402 (4 November 1993)

6 H.C. Dickenson and E.F. Mueller, J. Am. Chem. soc., 29 (1907), 1381

7 T.W. Richards and V. Yugve, J. Am. Chem. soc., 40 (1918), 164 